\title{
Comparative study of carcinoembryonic antigen and epithelial membrane antigen expression in normal colon, adenomas and adenocarcinomas of the colon and rectum
}

\author{
B R DAVIDSON*, V R SAMS**, J STYLES $\dagger$, C DEAN $\dagger$, P B BOULOS* \\ From the Departments of Surgery* and Histopathology**, University College and Middlesex School of \\ Medicine, London and The Institute of Cancer Research, Sutton, Surrey ${ }^{\dagger}$
}

SUMMARY The heterogeneous nature of tumour antigen expression may require selection of monoclonal antibodies on an individual patient or tumour basis to allow adequate tumour localisation. Carcinoembryonic antigen (CEA) and epithelial membrane antigen (EMA) expression has not previously been compared in colorectal cancer patients. Sections of cancer $(n=52)$, adjacent normal colon $(n=45)$, synchronous adenomas $(n=11)$ and nodal metastases $(n=49)$ were examined by indirect immunoperoxidase staining in 51 consecutive patients with colorectal cancer using monoclonal antibodies to CEA and EMA. The percentage of cells with positive staining in the primary tumours was graded 1: $<25 \%, 2: 25-49 \%, 3: 50-75 \%, 4>75 \%$. All primary colorectal cancers expressed CEA and 43 of 52 expressed EMA (83\%). Grading showed CEA greater than EMA in 39, equal in 11 and less in two. Well differentiated cancers were more frequently graded three or four for CEA staining (23 of 27) than moderately differentiated cancers (11 of 22$)(p<0 \cdot 01)$. Equivalent figures for EMA were four of 27 and three of 22 (not significant) (NS) although the majority (86\%) were graded 1 and 2 . Grade 1 CEA expression was found in six of 15 proximal and only two of 37 distal lesions ( $<<0.01, \chi^{2}$ test) while for EMA equivalent figures were three of 15 and six of 37 (NS). Nodal deposits all expressed CEA and 45 of 49 expressed EMA $(92 \%) ; 29$ of 45 normal colon sections showed CEA expression (64\%) as did all adenomas. EMA was not expressed by normal colon or adenomas. These results suggest that EMA expression is more specific but less sensitive than CEA for colonic cancer and is independent of tumour differentiation and site. Thus selecting monoclonal antibodies to CEA or EMA based on tumour biopsies may allow improved tumour localisation for imaging or therapy in patients with colorectal cancer.

Carcinoembryonic antigen (CEA) and epithelial membrane antigen (EMA) are both expressed by colorectal cancers. ${ }^{12}$ Monoclonal antibodies to these tumour associated antigens may have a role in diagnostic immunohistochemistry, ${ }^{34}$ in localisation of tumours and their metastases by radioimmuno-

Address for correspondence: Mr B R Davidson, Department of Surgery, University College and Middlesex School of Medicine, Rayne Institute, 5 University Street, London WC1E 6JJ.

Accepted for publication 24 October 1988. localisation ${ }^{5-7}$ and have potential for drug, radioisotope or toxin conjugated therapy..$^{8-10}$

The heterogenous nature of tumour antigen expression, however, may necessitate the use of more than one antibody whether used for diagnosis or therapy. ${ }^{11}$ In a previous study we have shown CEA and EMA expression in colorectal cancers and shown the presence of CEA but rarely EMA in adenomatous polyps and normal colon. ${ }^{12}$ The small numbers and random selection of tissues in this pre- 
liminary study, however, did not allow the antigenic expression to be adequately compared between cancers, nor between the primary cancer, normal colon and nodal metastases in individual patients. We have therefore examined CEA and EMA expression in a larger and consecutive series of specimens of colorectal cancer, their nodal metastases, synchronous adenomatous polyps and adjacent normal mucosa.

The association between CEA expression and tumour differentiation is controversial, with some studies showing greatest CEA expression in well differentiated $d^{131+}$ and others in poorly differentiated $^{1516}$ cancers. Moreover although CEA expression of cancers increases from proximal to distal in the gastrointestinal tract, ${ }^{17}$ a correlation between the site of colorectal cancers and their CEA expression has yet to be established. We have therefore also compared the CEA and EMA expression of cancers with their degree of differentiation and site.

\section{PATIENTS}

Fifty one consecutive patients undergoing potentially curative resection of a colorectal carcinoma in a single unit during a one year period were studied. There were 26 men and 25 women with a median age of 68 years (range 26-90).

\section{SPECIMENS}

A total of 52 colorectal cancers were studied, one patient having two synchronous carcinomas. All of the specimens were examined conventionally with haematoxylin and eosin staining (H\&E) before immunohistochemical examination, both procedures being carried out by independent investigators and the results subsequently being compared.

On conventional histopathological assessment six of the tumours were classified as Dukes stage A $(11 \cdot 5 \%), 26$ stage B $(50 \%)$ and 20 stage C $(38 \cdot 5 \%)$. Twenty seven tumours were graded as well differentiated $(52 \%), 22$ moderately differentiated $(42 \%)$ and three poorly differentiated $(6 \%)$. Fifteen of the primary tumours were in the right colon (caecum, ascending and right transverse) (29\%), 22 in the left colon (left transverse, descending and sigmoid) $(42 \%)$ and 15 in the rectum $(29 \%)$. The percentage of well and moderately differentiated cancers was similar in the right $(47 \%$ and $40 \%)$ and left $(55 \%$ and $41 \%)$ colon and in the rectum $(53 \%$ and $47 \%$ ) (all ns). On examination of 249 regional lymph nodes metastatic tumour deposits were found in 49 nodes from 20 patients. Eleven polyps were found in the resected specimens of which six were tubular and five tubulovillous adenomas.

Immunohistochemistry with anti-CEA and antiEMA was carried out using consecutive sections from tissue blocks which had been prepared for $H \& E$ staining. The expression of CEA and EMA was also examined in the adjacent normal colon in 45 of these patients $(88 \%)$; in the 11 adenomatous polyps found in the resected specimens and in the 49 lymph node metastases present in 20 specimens.

MONOCLONAL ANTIBODIES

Anti-CEA antibody C46 (Amersham, UK) is a mouse IgG monoclonal antibody raised to a high affinity sub fraction of CEA derived from the liver metastasis of a human colorectal carcinoma. ${ }^{18}$ AntiEMA antibody ICR2 is a rat $\mathrm{IgG}_{2 \mathrm{a}}$ monoclonal antibody raised using extracts of the human milk fat globule membrane (HMFGM) as immunogen (Institute of Cancer Research, Sutton, Surrey, UK). This antibody competitively inhibits the main epitope of the polyclonal antiserum previously raised to the $\mathrm{HMFGM}^{19}$ and has a similar distribution on screening of human tissues (Personal communication, J P Sloane, Institute of Cancer Research, Sutton, Surrey).

IMMUNOHISTOCHEMISTRY

All tissues had been formalin fixed, routinely processed and embedded in paraffin wax. Consecutive tissue sections were cut at $5 \mu$ and stained by the indirect immunoperoxidase staining method which was carried out as follows; after dewaxing in xylene and dehydrating in alcohol the endogenous peroxidase activity in the tissue section was blocked with a $0.5 \%$ solution of hydrogen peroxide in methanol. Both the anti-CEA and anti-EMA antibodies were applied at the same concentration $(10 \mu \mathrm{g} / \mathrm{ml})$ and for fixed time periods (one hour). The second antibody of the reaction was a 1:100 dilution of rabbit antimouse or rabbit antirat antibody conjugated to horseradish peroxidase (Dako, Uppsala, Sweden). The reaction product was identified using the chromogen diaminobenzidine and the sections counterstained with Mayers Haemalum. Positive control sections were colonic cancers known to express both antigens. Negative control sections consisted of either no first antibody (anti-CEA or anti-EMA) or neither first nor second antibodies being applied.

GRADING OF THE IMMUNOHISTOCHEMICAL STAINING

The staining of the primary tumours was assessed for both antibodies without knowledge of pathological or clinical details. Each slide was scanned at low power magnification $(x 10)$ and three randomly selected sites at high power magnification $(x 40)$. The percentage of tumour cells per field staining positively was assessed and scored on a four point scale based on the proportion of the total number of tumour cells: $<25 \%=$ grade $1,25-49 \%=$ grade $2,50-75 \%$ 


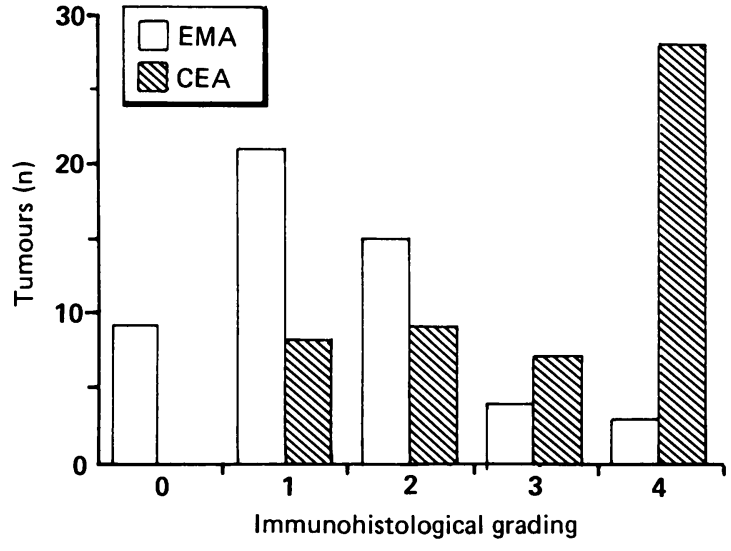

Fig. 1 Immunohistochemical grading of tumours. The tumours are categorised by the percentage of cells expressing either CEA of EMA into grades 1-4. The grade of immunohistochemical staining can be seen to be higher with the anti-CEA antibody than with the anti-EMA in the majority of tumours.

$=$ grade 3 and $>75 \%=$ grade 4 . A cell was considered positive if staining was present either intracellularly or on the membrane.

\section{Results}

PRIMARY TUMOURS

The staining pattern was similar for both the antiCEA and anti-EMA antibodies, being most pronounced on the luminal cell membrane in areas of tumour with gland formation. A cytoplasmic staining pattern was occasionally seen with both antibodies.

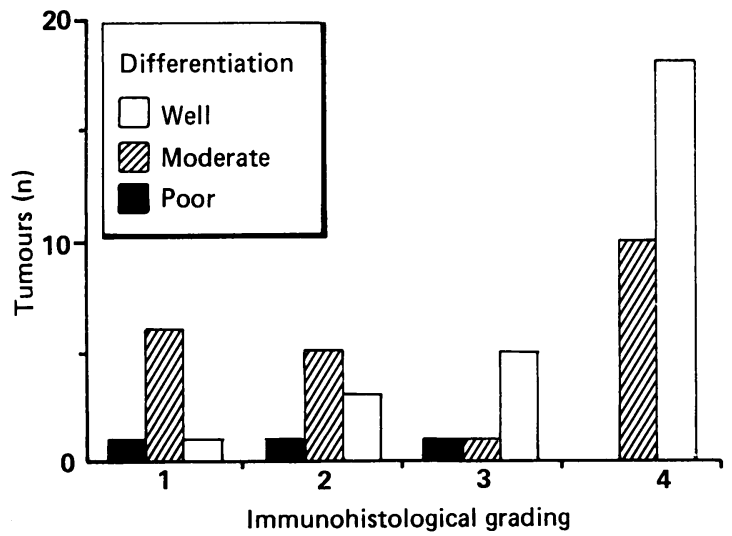

Fig. 2 CEA expression and tumour differentiation. The tumours are categorised by their immunohistochemical grading of CEA expression and degree of differentiation. Tumours expressing CEA in $>50 \%$ of cells (Grades 3 and 4) show a better grade of tumour differentiation.

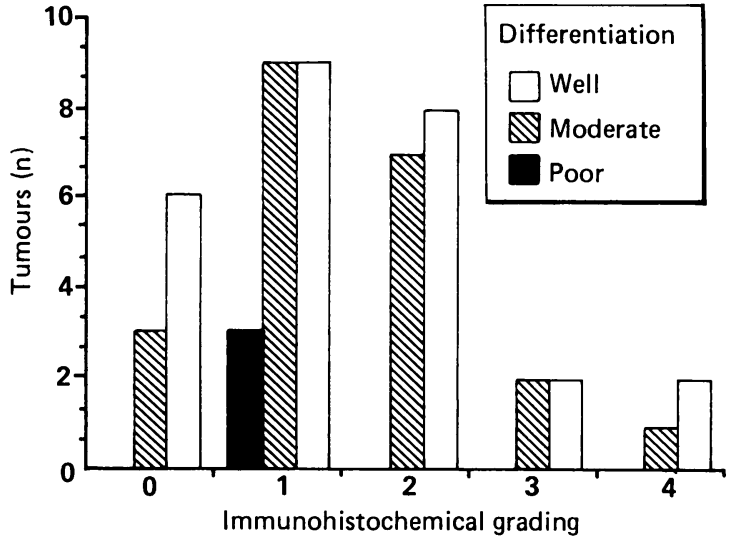

Fig. 3 EMA expression and tumour differentiation. Tumours have been categorised by their immunohistochemical grading of EMA expression and degree of tumour differentiation. In contrast to CEA no significant association was found between the EMA expression of the tumour and the degree of differentiation.

All 52 colorectal cancer sections stained positively for CEA whereas 43 sections stained positively for EMA $(83 \%)$. The grade of immunohistochemical staining of the tumours (Fig. 1) was higher with anti-CEA than anti-EMA in $39(75 \%)$, equal in $11(21 \%)$ and less in two $(4 \%)$. Tumours graded 3 and 4 for CEA expression were more commonly well rather than moderately differentiated (23 of $27 v 11$ of 22 , $\mathrm{p}<0.01, \chi^{2}$ test) (Fig. 2). No correlation was found between the staining pattern with anti-EMA and the degree of tumour differentiation (Fig. 3). Of the nine tumour samples with no detectable EMA expression three were moderately and six well differentiated.

Grading of CEA expression immunohistochemically varied with the site of the primary tumour, right sided colonic tumours showing less evidence of CEA expression than left sided. Six of the 15 right sided lesions were grade $1(<25 \%$ cell stain) whereas only two of the 37 lesions in the left colon and rectum were graded $1\left(\mathrm{p}<0 \cdot 01, \chi^{2}\right.$ test $)$ (Fig. 4). Equivalent figures for EMA expression were three of 15 in the right colon and 6 of 37 in the left colon and rectum (ns).

LYMPH NODES

All nodal metastatic deposits showed staining for CEA. Forty five of the 49 metastases from 20 cancer specimens stained positively for EMA $(92 \%)$, a similar proportion of positive staining than seen in the primary tumours from this group ( 19 of $20,95 \%$ ).

ADJACENT NORMAL COLON

A membranous pattern of staining involving the 


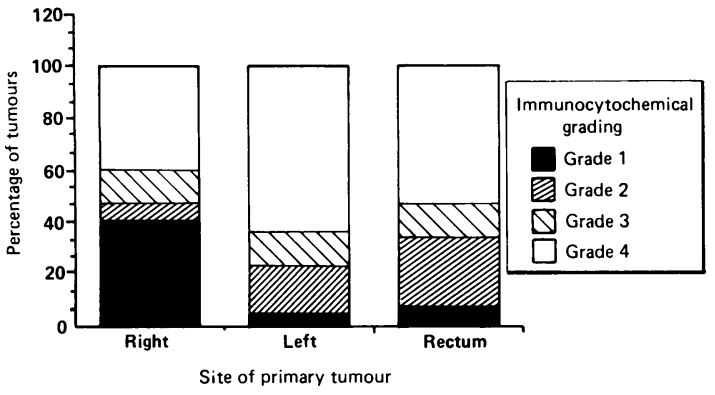

Fig. 4 CEA expression and site of tumour. The sites of the primary tumours have been divided into right colonic (ascending and right transverse colon), left colonic (left transverse, descending and sigmoid) and rectal and the percentage of tumours in each immunohistochemical grade is shown. Right sided colonic lesions show less evidence of $C E A$ expression than left sided. Six of the 15 right sided lesions were grade $1(<25 \%$ cell stain) whereas only two of the 37 lesions in the left colon and rectum were grade 1 $\left(p<0 \cdot 01, \chi^{2}\right.$ test $)$.

luminal surface of the colonic mucosa was found in 29 of the 45 specimens of normal colon $(64 \%)$ stained with the anti-CEA antibody whereas none of the sections showed evidence of EMA expression. There was no correlation between the CEA expression of normal colon and the immunohistochemical grading of the adjacent cancers. Of the 29 sections of normal colon expressing CEA 20 of the associated cancers were graded three or four for CEA expression $(69 \%)$, whilst for the 16 sections of normal colon which did not express CEA 11 of the adjacent cancers were graded 3 or $4(69 \%)$. The group of patients with both a colonic carcinoma and an adenomatous polyp more commonly expressed CEA in their adjacent normal colon than those with a carcinoma alone although this trend was not significant (eight of nine $(89 \%) v 21$ of $\left.36(58 \%), \chi^{2}, \mathrm{NS}\right)$.

ADENOMATOUS POLYPS

All of the polyps showed strong (grade 3-4) expression of CEA with a luminal membrane staining pattern similar to that of the normal colon adjacent to the tumour. None of the polyps expressed EMA.

\section{Discussion}

Clinical and pathological variables were compared between the present group of patients and those in a large prospective study on colorectal cancer. ${ }^{21121}$ Although the age distribution was similar the present group had a lower male to female ratio (26 to $51 v 506$ of $\left.709, \chi^{2}=9 \cdot 3, p<0.01\right)$ and a greater proportion of well $\left(27\right.$ of $52 v 203$ of $\left.709, \chi^{2}=12.4, \mathrm{p}<0.001\right)$ and a lower proportion of moderately ( 22 of $52 v 443$ of 709, $\left.\chi^{2}=8 \cdot 2, p<0 \cdot 01\right)$ differentiated cancers. There were no significant differences in the Dukes staging or the site of the cancers.

Our immunohistochemistry results further support the findings of previous smaller studies that CEA is expressed by all primary colorectal cancers and EMA in over $80 \% .^{2312}$

The grading system used has allowed cancers to be compared by their expression of tumour antigens. Although grading systems may be criticised for their semiquantitative nature the system selected for use in the present study has been successfully applied to the antigenic expression of breast cancers" and has been objectively assessed and found to be reproducible. ${ }^{.3}$ A correlation between CEA expression in colonic carcinomas and their degree of differentiation has previously been suggested, although reports as to whether CEA expression is greater in well or poorly differentiated tumours are conflicting. ${ }^{13-16}$ The grading has shown CEA expression to be greater in well rather than moderately differentiated cancers. Carcinoembryonic antigen expression in the present study was also greater in distal than proximal colonic cancers, which was not a reflection of differences in tumour differentiation by site. A similar increase in CEA expression has previously been noted in distal compared with proximal cancers in the gastrointestinal tract ${ }^{17}$ but scanty evidence exists of similar differences within the length of the colon. Normal tissue antigens such as mucins or blood group substances may either increase ${ }^{24}$ or decrease ${ }^{2526}$ from proximal to distal. There was no relationship, however, in the present study between the EMA expression of tumours and either their degree of differentiation or site.

A similar number of metastatic deposits and primary tumours expressed EMA. This conflicts with previous reports which suggested an enhanced expression of EMA in metastatic tumour deposits based on immunohistochemistry ${ }^{12}$ and by the imaging of metastases but not primary breast carcinomas after the administration of radiolabelled anti-EMA MAB M8 $8^{\circ}$.

The pattern of CEA expression found in normal colon was similar to that of adenomatous polyps with a linear staining of the luminal membranes. These results are consistent with other studies showing CEA to be a normal product of colonocyte differentiation and an extract of normal adult colon..$^{27}{ }^{2 \times}$ In this study EMA expression was not found in either the normal colon, which in all cases was sampled adjacent to the cancers, or in benign adenomatous polyps. This contradicts previous results suggesting either a weak positive staining of normal colon for $\mathrm{EMA}^{24}$ or its expression only in normal colon adjacent to neoplasia or inflammation. ${ }^{19}$ Such 
differences may be explained by the cross reactivity of polyclonal antisera used in previous studies compared with the specificity of the anti-EMA monoclonal antibody used in the present study. Of interest was the increased expression of CEA by the adjacent normal colon of patients in whom both a benign adenomatous polyp and colorectal cancer were present in comparison to those with a carcinoma alone. Although not statistically significant this trend may reflect a field change in the adjacent normal colon of these patients.

The antigenic expression of tumours and their metastases is vital information if antibodies to tumour associated antigens are to be considered for use in radioimmunolocalisation or for the targeting of radionuclides, drugs or toxins for therapeutic purposes. It is well recognised that tumour antigen heterogeneity is the rule rather than the exception" and in this study heterogeneity of antigen expression was noted with anti-CEA and anti-EMA within different portions of a given tumour and between primary and secondary tumours. From the present study we may conclude that CEA and EMA have a similar pattern of expression in primary colorectal cancers and their nodal metastases although EMA expression is more restricted than CEA. Normal colon and adenomatous polyps express CEA but not EMA.

This pattern of antigen expression is of clinical significance. Monoclonal antibodies to CEA or EMA selected for an individual patient or tumour based on immunohistochemical staining of biopsies may allow a greater antibody uptake in tumour tissue and improved tumour targeting for imaging or therapy in patients with colorectal cancer.

Mr B R Davidson gratefully acknowledges the financial support of the Wellcome Foundation.

\section{References}

1 Isaacson P, Le Vann PH. The demonstration of carcinoembryonic antigen in colorectal carcinoma and colonic polyps using an immunoperoxidase technique. Cancer 1976; 38: 1348-56.

2 Pinkus GS, Kurtin PJ. Epithelial membrane antigen - A diagnostic discriminant in surgical pathology. Hum Pathol 1985; 16: 929-40.

3 Heyderman E, Brown B, Richardson TC. Epithelial markers in prostatic, bladder and colorectal cancer; an immunoperoxidase study of epithelial membrane antigen, carcinoembryonic antigen and prostatic acid phosphatase. J Clin Pathol 1984; 37: 1363-9.

4 Mansi JL, Berger U, Easton D et al. Micrometastases in bone marrow in patients with primary breast cancer: evaluation as an early predictor of bone metastases. $\mathrm{Br}$ Med J 1987; 295: 1093-6.
5 Mach JP, Carrel S, Forni M, Ritschard J, Donath A, Alberto P. Tumour localisation of radiolabelled antibodies against carcinoembryonic antigen in patients with carcinoma. $N$ Engl J Med 1980; 303: 5-10.

6 Rainsbury RM. The localisation of human breast carcinomas by radiolabelled monoclonal antibodies. $\mathrm{Br}$ J Surg 1984; 71: 805-12.

7 Granowska M, Britton KE, Shepherd JH et al. A prospective study of ${ }^{123}$ I labelled monoclonal antibody imaging in ovarian carcinoma. J Clin Oncol 1986; 4: 730-6.

8 Ballantyne KC, Perkins AC, Pimm MV, et al. Biodistribution of a monoclonal antibody-methotrexate conjugate (791T/36-MTX) in patients with colorectal cancers. Int J Cancer 1988; suppl 2: 103-8.

9 Casselas P, Brown JP, Gros O, et al. Human melanoma cells can be killed in vitro by an immunotoxin specific for melanoma associated antigen p97. Int J Cancer 1982; 30: 437-43.

10 Kalofonos HP. Stewart S, Epenetos AA. Antibody guided diagnosis and therapy of malignant lesions. Int $J$ Cancer 1988; suppl 2: 74-80.

11 Edwards PAW. Heterogenous expression of cellsurface antigens in normal epithelia and their tumours, revealed by monoclonal antibodies. Br J Cancer 1985 ; 51: $149-60$.

12 Davidson BR, Yiu CY, Styles J, Ormerod M, Clark CG, Dean C. A comparison of carcinoembryonic antigen (CEA) and epithelial membrane antigen (EMA) in human colorectal cancer. Int J Cancer 1988; suppl 3: 56-60.

13 Denk H. Tappeiner G, Eckerstorfer R, et al. Carcinoembryonic antigen (CEA) in gastrointestinal and extragastrointestinal tumors and its relationship to tumour cell differentiation. Int J Cancer 1972; 10: 262-72.

14 Goldenberg DM, Sharkey RM, Primus FJ. Carcinoembryonic antigen in histopathology: Immunoperoxidase staining of conventional tissue sections. $J$ Natl Cancer Inst 1976: 57: 11-22.

15 Rognum T, Elgjo K, Brandtzacg P, Orjasaeter $H$, Bergan A. Plasma carcinoembryonic antigen concentrations and immunohistochemical patterns of epithelial marker antigens in patients with large bowel carcinoma. J Clin Pathol 1982; 35: 922-33.

16 Bordes M. Michacls R, Martin F. Detection by immunoflourescence of carcinoembryonic antigen in colonic carcinoma, other malignant or benign tumours, and non-cancerous tissues. Digestion 1973; 9: 106-15.

17 Gold P, Freedman SO. Demonstration of tumourspecific antigens in human colonic carcinoma by immunological tolerance and adsorption techniques. $J$ Exp Med 1965; 121: 439-62.

18 Rogers GT, Scarle F, Bagshawe KD. Carcinoembryonic antigen: Isolation of a sub-fraction with high specific activity. Br J Cancer 1976; 33: 357-62.

19 Heyderman E, Steel K. Ormerod MG. A new antigen on the epithelial membrane; its immunoperoxidase localisation in normal and neoplastic tissuc. J Clin Pathol 1979; 32: 35-39.

20 Chapuis PH, Dent OF, Fisher R, et al. A multivariate analysis of clinical and pathological variables in 
prognosis after resection of large bowel cancer. $B J$ Surg 1985; 72: 698-702.

21 Payne JE, Chapuis PH, Pheils MT. Surgery for large bowel cancer in people aged 75 years and older. Dis Col Rectum 1986; 29: 733-7.

22 Ellis IO, Hinton CP, MacNay J, et al. Immunocytochemical staining of breast carcinoma with the monoclonal antibody NCRC-11: a new prognostic indicator. $\mathrm{Br}$ Med J 1985; 290: 881-3.

23 Muir IM, Ellis IO, Bell J, Robins RA. NCRC-11 Immunoperoxidase staining patterns in breast cancer; interpretive and technical reproducibility. Histopathology 1987; 11: 1208-10.

24 Nairn RC. Fothergill JE, McEntegart MG, Porteous IB. Gastro intestinal specific antigen: An immunohistological and serological study. $B r \operatorname{Med} J$ 1962; i: 1788-90.

25 Hartmann G. Investigations in group antigens in secretions and in aqueous extracts of organs. In: Group antigens in human organs, Copenhagen: Munsksgaard, 1970: 135-84.

26 Hounsell EF, Feizi T. Gastrointestinal mucins. Med Biol 1982; 60: 227-36.

27 Ahnen DJ, Kinoshita K, Nakane PK, Brown WR. Differential expression of carcinoembryonic antigen and secretory component during colonic epithelial cell differentiation and in colonic carcinomas. Gastroenterology 1987; 93: 1330-8.

28 Khoo SK, Warner NL, Lic JT. Mackay IR. Carcinoembryonic antigen activity of tissue extracts: a quantitative study of malignant and benign neoplasms. cirrhotic liver, normal adult and fetal organs. Int $J$ Cancer 1973: 11: 681-7.

29 Sloane JP. Ormerod MG. Distribution of epithelial membrane antigen in normal and neoplastic tissues and its value in diagnostic tumour pathology. Cancer 1981; 47: 1786-95. 